\title{
KONZEPTE
}

\section{Gelingendes Management}

\author{
Ingolf Hübner und Uwe Schwarzer
}

In der Sozialwirtschaft gelten heute für das Überleben von Unternehmen weitgehend die gleichen Regeln wie auf privaten Märkten. So unterscheidet sich auch Management in der Diakonie nicht grundsätzlich von anderen Formen verantwortlicher und professioneller Leitung von Unternehmen. Sie unterscheidet sich aber teilweise in den von ihr gemachten Voraussetzungen und ihren Ergebnissen sowie der Auswahl, Weiterentwicklung und Anwendung von Methoden, Instrumenten und Werkzeugen.

Managementstile sind kulturell geprägt und Managementstile prägen Kulturen. Der Einfluss sozialer Kontexte, Wertüberzeugungen, tradierte Organisationsformen und Verhaltensweisen können in einem Kulturbegriff zusammengefasst werden.

Hier ist kein enger Kulturbegriff als Oppositionsbegriff beispielsweise $\mathrm{zu}$ »Natur « oder zu »Massenkultur « gemeint, von dem andere Bereiche als nicht kulturell unterschieden werden. Von einem weiten Kulturbegriff wird das alltägliche Handeln in seiner Gesamtheit erfasst. Bei dem Versuch, einen solchen Kulturbegriff material zu füllen und nach seiner Interdependenz zu Managementstilen zu fragen, wird deutlich, dass Kultur drei wesentliche Bezüge aufweist. Kultur bezieht sich erstens auf Einstellungen und innere Haltungen. Kultur bezieht sich zweitens auf Handlungen und individuelle Verhaltensweisen. Kultur bezieht sich drittens auf Organisationsformen mit ihren rechtlichen, ökonomischen und strukturellen Festlegungen.

Diese unterschiedlichen Bezüge bringen es mit sich, dass einerseits typische Verhaltensweisen und Organisationsformen beobachtet und beschrieben werden können. Andererseits sind aber Überzeugungen und innere Haltungen prägend, die sich zumindest teilweise der Beobachtung entziehen. Diese Differenz wirkt sich insbesondere in Kontexten aus, die normativ geprägt sind. Die normativen Überzeugungen äußern sich in Positionierungen und Aktionen, aber sie gehen nicht darin auf. Auch im Management werden Überzeugung und Handlung selten übereinstimmen. Und dennoch gehören beide Seiten zur Verantwortung im Management. Sowohl Überzeugungen als auch Handlungen korrespondieren mit kulturellen Umfeldern.

Der enge Zusammenhang von christlichem Glauben und sozialem Engagement ist der Ausgangspunkt diakonischer Kultur. Diakonische Kultur ist Lebenspraxis und Gestaltungsaufgabe im christlichen Sinnhorizont. Dadurch unterscheidet sich diakonische Kultur nicht grundsätzlich

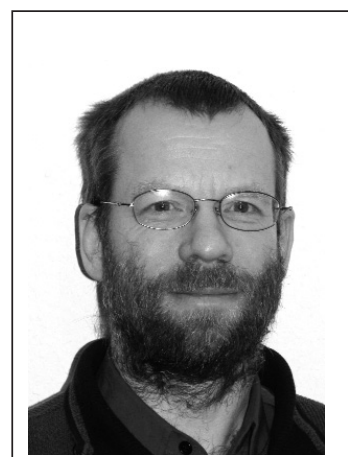

Dr. Ingolf Hübner (51) ist Diplomingenieur für Energieanlagentechnik und Theologe. Seit 1995 ist er im Diakonischen Werk der EKD in verschiedenen Funktionen tätig. Derzeit befasst er sich in der Stabsstelle Theologie beim Vorstand des Diakonischen Werkes der EKD mit diakoniewissenschaftlichen, sozialethischen und bioethischen Fragen.

E-Mail huebner@diakonie.de

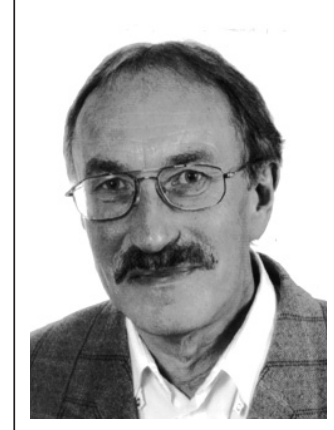

Uwe Schwarzer (52) ist Sozialund Verwaltungswissenschaftler, EQA-Assessor, TQM-Systemauditor. In der Stabsstelle Strategisches Management beim Vorstand des Diakonischen Werkes der EKD beschäftigt er sich mit Fragen des Managements und des Qualitätsmanagements, mit Verbraucherschutz und innovativen Sozialprojekten. E-Mailschwarzer@diakonie.de

von anderen Formen verantwortlich gelebter Lebenspraxis. Sie unterscheidet sich aber teilweise in den von ihr gemachten Voraussetzungen und teilweise in ihren Ergebnissen. Zu den Voraussetzungen gehört, dass Diakonie sich als praktische Auswirkung des christlichen Glaubens versteht. Diese Perspektive unterstreicht den weiten Kulturbegriff, da christlich gesehen Kultur auf die Gesamtentfaltung menschlicher Persönlichkeit, das Wohl der Gemeinschaft und die Verwirklichung einer humanen Gesellschaft ausgerichtet ist. Diese Perspektive beinhaltet aber auch die Warnung an das Management, dass Kultur kein erzeugbares Produkt ist, sondern als Gestaltungsraum von dem ihn prägenden Geist abhängt.

Management innerhalb kirchlicher wie diakonischer Strukturen muss dem Anspruch des Evangeliums gerecht werden. Das bedeutet, eigene Gaben und Fähigkeiten als Geschenk anzunehmen. Zugleich kann auch im Management Beruf nicht mit Berufung gleichgesetzt werden. Auch Führen und Leiten muss erlernt werden wie andere Berufe. Diese Differenzierung, die in der Theologiege- 
schichte als Verhältnisbestimmung von Person und Amt diskutiert wurde, erlaubt auch für das Management eine Unterscheidung zwischen persönlichen Anlagen und funktionalen Kompetenzen. Als Person sind auch Menschen mit Managementverantwortung Geschöpfe Gottes, die angenommen und geliebt sind und zugleich auf Gottes Vergebung angewiesen sind. Daraus folgt eine Relativierung von Macht. Kompetenzen und das funktionale Zusammenwirken vieler in Institutionen sind auf gegenseitige Ergänzung angelegt. Management in einer diakonischen Kultur soll — nach Möglichkeit partnerschaftliche - Beteiligung, Transparenz, Nachvollziehbarkeit, (strukturelle) Klarheit, dialogische und klare Kommunikation, Integration, Gerechtigkeit, Wertschätzung und Akzeptanz gewährleisten. In der Diakonie geht es deshalb neben einem guten Management im Sinne eines handwerklichen Handelns vor allem um Führung. Der Unterschied zwischen Führung und Management kann auf die Formel gebracht werden: Manager machen die Dinge richtig (analysieren, planen, Ziele setzen, kontrollieren etc.), Führende machen die richtigen Dinge (initiieren, motivieren, coachen etc.).

Zum Führen gehört es, Vorbild zu sein, die Fähigkeiten der Anderen zu erkennen und zu fördern sowie die Mitarbeitenden zu motivieren. Management in einer diakonischen Kultur ist vor dem Hintergrund seiner spezifischen normativen Ausrichtung allem ein personales und gemeinschaftsbezogenes Geschehen.

\section{Ganzheitlich denken und handeln}

Ein diakonisches Unternehmen im Jahre 2007 muss als ein komplexes System verstanden werden: Viele Elemente gehören zu dieser Ganzheit eines Systems.

Wenn »Bewahrung der Schöpfung « in der Diakonie heißt, alle Aspekte der Schöpfung zu berücksichtigen, so gehört neben der Sorge für Menschen in Not auch die Verantwortung gegenüber der Gesellschaft, der Natur, der Technologie und der Wirtschaft dazu. Bei der Bereitstellung eines differenzierten Angebotsspektrums sozialer Dienstleistungen ist dabei nicht nur der sogenannte »Kunde« von Bedeutung, sondern auch die Mitarbeiterschaft, die Kostenträger, der Staat in seiner sozialen Verantwortung, die Lieferanten als Koproduzenten unserer diakonischer Leistungen sowie auch die Konkurrenz mit ihrer jeweiligen Werteorientierung.

In einem solchen komplexen System ist es deshalb nur folgerichtig, diakonisches Management als ein integriertes Management zu verstehen. Angesichts der Anliegen und Interessen der Diakonie, ihrer Überzeugungen und Werte sowie auch ihrer Ressourcen, ist unter Management nicht etwa nur die Funktion von Führungskräften zu verstehen, sondern ein System von Aufgaben, die von der gesamten Mitarbeiterschaft gestaltet, gelenkt und weiterentwickelt wird.

\section{Managementmodelle und Anspruchsgruppen}

Die Wahl eines entsprechenden Managementmodells kommt in der Diakonie besonderer Bedeutung zu. Abgeleitet wird das Modell aus der Orientierung an den Wün- schen und Bedürfnissen von Menschen in besonderen Lebenslagen: Im Blick auf die sogenannte Kundenorientierung werden in diakonischen Einrichtungen bei der strategischen Unternehmensführung bestimmte Anspruchsgruppen-Konzepte favorisiert, andere eher nicht verwendet wie etwa:

\section{- der Shareholder-value-Ansatz (Gewinnmaximierung)}

- der Stakeholder-value-Ansatz (langfristig ausgewogene Berücksichtigung aller Anspruchsgruppen zur Erreichung eines maximalen Shareholder-values).

Das ethische (normativ-kritische) Anspruchsgruppenkonzept findet in der Diakonie häufig Anwendung: Als relevante Anspruchsgruppen gelten Menschen, deren Men-

\section{"Was bleiben will, muss sich ändern «}

schenwürde zu achten ist und deren moralische Rechte zu stärken sind. Es geht also nicht um die Befriedigung jener Gruppen, die am wirkmächtigsten sind und deren Ansprüche befriedigt werden müssen, sondern um die ethisch begründbare Legitimität (z. B. gegenüber den Schwachen in einer Gesellschaft).

Nicht alle Managementmodelle erfüllen diese Erwartungen der Diakonie. Zwei in der Diakonie häufig angewandte Modelle sind das neue St. Galler-Managementmodell, das vielen Leitungskräften der Diakonie an der Universität St. Gallen vermittelt wurde sowie das Modell der European Foundation of Quality Management, ein Modell, das einem diakonischen Unternehmen zudem den Weg zu einem europäischen Business excellence ebnet. Die Managementqualität von diakonischen Trägern, die diese Modelle umsetzten, erfuhr die Wertschätzung branchenübergreifender Bewertungen wie beispielsweise durch das Institut der deutschen Wirtschaft (Samariter-Stiftung Nürtingen als EFQM-Anwender).

Management in der Diakonie benötigt eine exzellente Leitung. Derzeit wird im Diakonischen Werk der EKD ein Curriculum zur (Weiter-) Qualifizierung von Vorständen und Aufsichtsräten erarbeitet, das in Fortbildungsangeboten der neu gegründeten Führungsakademie umgesetzt werden soll.

Auf der Basis von Leitbild und Managementmodell haben viele diakonische Unternehmen Managementkonzepte und Managementstrategien entwickelt, die das vorrangige Ziel haben, die Vision einer Einrichtung in ein zukunftsorientiertes und diakonisch profiliertes Handeln umzusetzen. Zur Steuerung der Organisation werden dabei beispielsweise Managementprinzipien wie Seelsorgekonzept, Projektmanagement, Zielvereinbarungen auf allen Ebenen, Funktionen-Diagramme, Personalentwicklung-, Führungs- und Kommunikationskonzepte erarbeitet. Im Rahmen des strategischen Managements werden aus den strategischen Zielen und unter Nutzung etablierter Konzepte - wie beispielsweise Controllingkonzepte - 
letztlich Strategieumsetzungsprozesse initiiert und bewertet.

Nicht nur die Sichtung adäquater Geschäftsmodelle, sondern auch die Auswahl und die Weiterentwicklung von Managementinstrumenten und Managementwerkzeugen spielen bei den normativ-ethischen Ansätzen der Diakonie eine große Rolle. Die Anwendung der Balanced Scorecard ist in der Diakonie zu einem Standard geworden, wenn es darum geht, Leitbilder und Visionen in konkrete Handlungen und Aktionen zu transformieren. Liegen die strategischen Grundlagen wie die Vision und das Leitbild, die Unternehmensziele, die Unternehmenskultur und Unternehmensethik vor, so lassen sich mit dem Instrument der Balanced Scorecard Strategiekonzepte mit Portfolio, Entwicklungsrichtungen, Standortbestimmungen sowie Zielen und Maßnahmen erarbeiten. So gelingt es mit der Balanced Scorecard die oftmals sehr abstrakt formulierten Leitbilder und Strategien so zu konkretisieren, dass deren Umsetzung zielgerichtet gesteuert werden kann.

Einige diakonische Unternehmungen gehen einen weiteren Schritt, indem sie Managementinstrumente diakoniebezogen fortentwickeln. Dies kann gerade am Beispiel der Balanced Scorecard verdeutlicht werden: In der von Bodelschwinghschen Anstalt Bethel wurde die Balanced Scorecard ergänzt und über die fünf Perspektiven Kunden/Adressen,

Finanzen, Mitarbeitende, Prozesse/Strukturen und Unternehmensentwicklung hinaus auch Ziele zur diakonischen Identität und zu Werten formuliert. Das Ziel, das ein christliches Selbstverständnis das Leben und Arbeiten prägt, wurde in das Berichtswesen und Controlling aufgenommen.

In der diakonischen Praxis angewandt werden auch selbst entwickelte (ethisch-normative) arbeitsfeldspezifische Managementinstrumente: So dient das in der Behindertenarbeit entwickelte GBM (EDV-gestütztes Verfahren zur Gestaltung der Betreuung von Menschen mit Behinderungen) sowohl als ein Führungsinstrument für die strategische und betriebliche Ebene einer Einrichtung als auch ein Instrument der Qualitätssicherung.

\section{Qualitätsmanagement}

Qualitätsmanagement spielt in der Unternehmenskultur der Diakonie eine große Rolle. Diakonische Arbeit darf nicht nur gut gemeint, aber unprofessionell und qualitativ fragwürdig sein. Deshalb leiten sich aus der Beschreibung diakonischer Qualität Strategien, Strukturen und Prozesse ab. Das »Handbuch Diakoniesiegel Pflege« macht anschaulich deutlich, dass es nicht einfach darum geht, Qualitätsnormen wie die DIN EN ISO zu übernehmen. Es sind theologische Leitsätze, die Ausgangspunkt diakonischer Qualitätsdefinitionen sind. Obwohl der Begriff der DIN EN ISO in dem Handbuch selbst nicht vorkommt, werden die Leitsätze so operationalisiert, dass mit den etablierten Instrumenten und Werkzeugen des Qualitätsmanagements Standards diakonischer Arbeit aufgezeigt werden, die dann allerdings durchaus ISO-Anforderungen erfüllen und von unabhängigen Zertifizierern bestätigt werden.
Christliche Wertüberzeugungen sind der Ausgangspunkt für die Standardsetzung. Nach christlichem Verständnis sind Menschen zur Bewahrung der Schöpfung aufgerufen. Dieses verpflichtet zu einem verantwortlichen Umgang mit Ressourcen. Dies trifft auf die ökologischen Ressourcen unserer Erde ebenso zu, wie auf die zur Aufrechterhaltung und Weiterentwicklung der sozialen Dienstleistungen notwendigen finanzwirtschaftlichen, personalwirtschaftlichen und organisatorischen Grundlagen. Am Ende dieser Operationalisierungsprozesse stehen dann die Einhaltung von Standards Sozialer Arbeit, ökonomischer Standards (Controlling, Kennzahlen) sowie auch ökologischer Standards wie beispielsweise der EMASStandard Umwelt im Diakonischen Werk der EKD.

Es gibt viele Beispiele dafür, wie in der Diakonie Methoden, Instrumente und Werkzeuge des Managements benellen Wohlfahrtsverbandes zu erfüllen. Ein Beispiel: Gelebte Spiritualität hat in diakonischen Einrichtungen einen hohen Stellenwert. So wird in der Einrichtung Herzogsägemühle das Instrumentarium des Qualitätsmanagements am Beispiel der Qualitätszirkelarbeit so genutzt, dass die in der Einrichtung gelebte Spiritualität den Wünschen und Bedürfnissen der Mitarbeitenden entspricht.

Instrumente des Qualitätsmanagements werden genutzt, um im Rahmen einer Dienstgemeinschaft größtmögliche Transparenz und Partizipation zu ermöglichen. In der Theodor-Fliedner-Stiftung im Mühlheim wurde ein virtuelles Qualitätsmanagement-Handbuch entwickelt. Dieses Handbuch ist nicht nur allen Mitarbeitenden zugänglich, sondern kann - im jeweiligen Verantwortungsbereich - von den einzelnen Mitarbeitenden jeweils auch so verändert und ständig verbessert werden, dass Strukturen und Prozessabläufe einer ständigen Optimierung unterliegen.

Bei der Verfolgung des Ziels, den Menschen in Not ins Zentrum allen Handelns zu stellen, ist in der Diakonie die Erkenntnis gewachsen, dass die Darstellung von Ergebnisqualität sozialen Handelns nicht ausreichend ist. Viele diakonische Einrichtungen sind dazu übergegangen mit Wirkungsanalysen und der Beschreibung von UrsacheWirkungsbeziehungen stärker die sogenannte OutcomeQualität (anstelle von output) zu beschreiben, die bei den Menschen in ihrer Hilfesituation die größte Wirkung erzeugt. So haben die Zieglerschen Anstalten der Diakonie sehr bewusst die Ursache-Wirkungsbeziehungen in Verbindung gebracht mit den definierten strategischen Zielen. Dabei wurde deutlich, dass Ursache-Wirkungsketten auch einen sehr starken kommunikativen Faktor haben: Sie schaffen bei der Führungsebene Bewusstsein über die Zusammenhänge und Bedeutung verschiedener Ziele und fördern das gemeinsame Verständnis der gesamten Managementstrategie. So soll dargestellt werden, wie die unterschiedlichen Bereiche (Potenziale, Prozesse, Kunden, Finanzen) zusammenwirken müssen, um die Manage- 
mentstrategie umsetzen zu können. So soll das Ziel erreicht werden, dass die Qualität diakonischer Dienstleistungen und die Erfüllung des diakonischen Auftrages gesichert werden auf der Basis eines tragfähigen wirtschaftlichen Fundaments. Damit haben wirtschaftliche und soziale Belange bei den Unternehmenszielen eine gleich hohe Bedeutung. Die Berücksichtigung ökonomischer und ökologischer Ziele unterstreicht den Anspruch eines nachhaltigen Handelns.

Wer über Management in der Diakonie nachdenkt, darf die aktuelle Diskussion über die Höhe der Managementgehälter nicht außen vor lassen. Die Evangelische Kirche in Deutschland hat deutlich gemacht, dass unverhältnismäßig hohe Gehälter von Managern das Vertrauen der Menschen in die Wirtschaft zerstören. Der Abstand zwischen Gehältern in einem Unternehmen muss vor den Beziehern der geringsten Gehälter gerechtfertigt werden können. Der Diakonische Coporate Governance Codex verpflichtet als Operationalisierungsvorschlag deshalb die Leitungen diakonischer Unternehmen, Transparenz über die Vorstandsgehälter herzustellen.

\section{Resümee}

»Was« an sozialen Dienstleistungen erbracht werden soll, bestimmt der »Kunde« und dies immer wieder neu. »Wie« dies zu geschehen hat unterliegt dem Management dieser Dienstleistungs- und Veränderungsprozesse.

Die in der Diakonie angewandten Managementmodelle und Methoden sind von einer Kultur geprägt, bei der alle Handlungsvollzüge vom christlichen Glauben und der Hoffnung darauf beeinflusst sind, dass allen Menschen Gottes Heil zuteil wird. Gleichzeitig geben die in der Arbeit angewandten und zum Teil auch selbst entwickelten Managementinstrumente der Diakonie selbst ihr typisches Profil.

Beschreibungen eines diakonischen Managements sind jedoch nur Momentaufnahmen. Diakonische Kultur entwickelt sich weiter. So wie auch die sich ständig ändernden Lebenslagen der Menschen und auch die sozialpolitischen Rahmenbedingungen. Das Management der Diakonie muss sich immer wieder neu diesen Veränderungen stellen und sich auch selbst immer wieder erneuern, so wie es das Motto einer großen diakonischen Einrichtung auszudrücken versucht: »Was bleiben will, muss sich ändern. Unternehmen statt unterlassen.«

\section{Grundlegendes zum Management in der Diakonie}

Der Management-Stil der Diakonie ist das Ergebnis der Transformation dieser Grundanliegen in konkretes Managementhandeln. Diese Operationalisierung geschieht in verschiedenen Stufen und anhand unterschiedlicher Modelle, Konzepte, Methoden und Werkzeuge. Hierzu haben die Diakonie sowie die Evangelische Kirche in Deutschland eine Reihe von Erwartungen und Selbstverpflichtungen formuliert, die im Prozess der Operationalisierung gemeinhin als sogenannte Vorgabedokumente bezeichnet werden:

- Die EKD-Denkschrift »Unternehmerisches Handeln in evangelischer Perspektive« macht Empfehlungen für verantwortliches wirtschaftliches Handeln in Deutschland, skizziert einen umfassend nachhaltigen Wirtschaftsstil und beschreibt letztendlich eine »Unternehmerethik« wie sie auch für das Management der Diakonie von Bedeutung ist. Dazu zählt insbesondere auch das Engagement im Bereich »Corporate social responsibility«, das nach Aussage von Unternehmensberatern weit langlebiger sein wird als aktuelle Managementmethoden oder

Renditemaximierungsstrategien (Ernst und Young CSR-Newsletter April 2007).

- Der Diakonische Corporate Governance Kodex (DGK 2005) orientiert sich an der Stärkung diakonischer Einrichtungskultur sowie der Optimierung der Leitung und Überwachung diakonischer Einrichtungen und gibt Standards und Empfehlungen zu guter Einrichtungsführung.

- Die Verbindung zwischen geistlicher und kirchlicher Gemeinschaft sozialer Verantwortung und Organisation von Hilfeleistungen wird in dem Dokument »Charakteristika einer diakonischen Kultur» ebenso dargestellt wie Anforderungen an Führung und Leitung sowie Wirtschaften und Haushalten in der Diakonie.

- Die sogenannte Loyalitätsrichtlinie der EKD formuliert die beruflichen Anforderungen an die Leitung von Einrichtungen, an die Grundlagen eines kirchlichen Dienstes sowie die Anforderungen an privatrechtliche Dienst- und Arbeitsverhältnisse.

- Nicht zuletzt versucht das »Leitbild Diakonie Damit Leben gelingt « die Grundlagen für ein gelingendes Zusammenarbeiten, Zusammenleben und damit auch gelingenden Leitungs- und Managementhandeln zu beschreiben. 